SLAC-PUB-7888

hep-ph/9807511

July 1998

\title{
Atmospheric $\nu_{\mu}$ Deficit from Decoherence
}

\author{
Yuval Grossman and Mihir P. Worah \\ Stanford Linear Accelerator Center \\ Stanford University, Stanford, CA 94309
}

\begin{abstract}
The simplest explanation for the observed deficit of atmospheric muon neutrinos is that they have oscillated into tau or sterile neutrinos with an oscillation length of the order of the Earth diameter. In order to confirm this hypothesis, all other possible explanations should be ruled out. We propose that a viable alternative hypothesis is that the muon neutrino deficit is caused by flavor sampling events that result in a loss of coherence. The coherence length of the muon neutrinos is expected to be approximately one Earth diameter. We give predictions and experimental tests of this scenario.
\end{abstract}

\section{Submitted to Physics Letters B}

Research supported by the Department of Energy under contract DE-AC03-76SF00515 
The recent data on the flux of atmospheric neutrinos reported by the Super-Kamiokande (SuperK) collaboration gives strong evidence for neutrino mass [1]. The old data that indicated that the integrated ratio of muon like events to electron like events is smaller than expected was confirmed. Moreover, this ratio was shown to depend on the zenith angle and on the energy of the incident neutrinos. In particular, no evidence for disappearance was found for down coming events, where the neutrinos travel short distances. While for up coming events, where the neutrino travel much longer, the number of muon events was about half of the expected number. Furthermore, a depletion in the expected number of muon events as a function of $(L / E)$, where $L$ and $E$ are the inferred flight length and energy of the neutrino, was found.

The simplest explanation for the disappearance of the muon neutrino $\left(\nu_{\mu}\right)$ is that it has oscillated into a tau neutrino $\left(\nu_{\tau}\right)$ or a sterile neutrino $\left(\nu_{s}\right)$ with an oscillation length which is comparable to the Earth diameter. The best fit to the data indicates mixing with a mass squared difference $\Delta m^{2}=O\left(10^{-3}\right) \mathrm{eV}^{2}$, and a vacuum mixing angle $\sin ^{2} 2 \theta=1$. There exist other less likely explanations for the $\nu_{\mu}$ deficit that need to be ruled out based on the data itself in order to undoubtedly confirm the simple and elegant oscillation hypothesis. One is that the $\nu_{\mu}$ decays between its production in the atmosphere and its detection on the earth [2]. Another is that a violation of Lorentz invariance or a breakdown of the equivalence principle results in $\nu_{\mu}$ disapperance [3].

In this note we postulate another speculative mechanism that seems to agree with the data. We propose that the $\nu_{\mu}$ coherence length is somewhat less than the diameter of the Earth. This coherence loss is due to some unknown mechanism that measures the neutrino flavor. $^{a}$ As a simple example consider the case of $\nu_{\mu}-\nu_{s}$ mixing. In this scenario, the down going $\nu_{\mu}$ 's travel a distance much shorter than their coherence length, and hence remain $\nu_{\mu}$ 's. The up going $\nu_{\mu}$ 's, however, travel a distance of a few times their coherence length, and hence are reduced to the equilibrium state containing an equal amount of $\nu_{\mu}$ and $\nu_{s}$. Since, at present, there is no evidence that flavor remains coherent over distance scales larger than a few kilometers, it is important to rule out this mechanism in order to establish the neutrino oscillations hypothesis and its parameters.

With standard model interactions the coherence length for neutrinos of a few GeV energy is several orders of magnitude larger than the earth diameter. Therefore, in order for decoherence to explain the atmospheric neutrino deficit one needs a new mechanism of flavor dependent coherence loss for the neutrinos. (It must be flavor dependent in order to leave the atmospheric electron neutrinos unaffected.) Some examples of scenarios that could result in such a decoherence are a very large neutrino background [4], flavor dependent interaction into an extra dimension [5], or even small violations of quantum mechanics. We do not explore any of these ideas in detail since our purpose is to concentrate on the general features of the decoherence explanation and, in particular, on its experimental tests.

Neutrino propagation with coherence loss for the two species case can be described by the equation [6]:

${ }^{a}$ Coherence loss due to wave packet separation does not measure the flavor of the propagating state and is irrelevant here. 


$$
\frac{d \mathbf{P}}{d t}=\mathbf{v} \times \mathbf{P}-D \mathbf{P}_{T}
$$

Here $\mathbf{P}$ is a "polarization" vector such that $\mathbf{P}_{z}=+1(-1)$ corresponds to a pure $\nu_{\mu}\left(\nu_{s}\right)$. $\mathbf{P}_{T}$ is the transverse part of $\mathbf{P}, \mathbf{v}$ is the mass eigenstate vector such that $|\mathbf{v}|=\Delta m^{2} / 2 E$, and it is an angle $2 \theta$ from the $z$ axis, and $D$ is the (energy dependent) damping coefficient [6]. While the general solution to the above formula is complicated, we can understand its implications in several limiting cases. The weak damping limit, $D \ll|\mathbf{v}|$, corresponds to the large $\Delta m^{2}$ limit where the oscillations are too rapid to be observed, and must be averaged over. In this case the $\nu_{\mu}$ survival probability is given by

$$
P_{\mu \mu}(t)=\frac{1}{2}\left[1+\cos 2 \theta e^{-t / \tau(E)}\right], \quad \tau(E)^{-1}=D(E) \sin 2 \theta \sqrt{1-\frac{\sin ^{2} 2 \theta}{4}} .
$$

In the case of critical damping $(D \sim|\mathbf{v}|)$, the damping time is similar as above, however the oscillations will not average out, and the survival probability will be more complicated. Finally in the over damped case, the relaxation time is much longer [6] and is not of relevance here.

We concentrate on the weakly damped case given by Eq. (2) and propose that this is the form the muon survival probability at SuperK should be fitted for. While we cannot perform a fit of Eq. (2) to the data, it seems it can agree with it. The mean free path needed to explain the SuperK data should be somewhat smaller than the Earth's diameter, $\tau \sim 10^{-2} \mathrm{~s}$, where $\tau$ is presumably a smooth function of the energy. Moreover, in order to not significantly deplete the downward going neutrinos, we require $\sin 2 \theta \lesssim 0.4$. Then, the suppression for the up going muons is about a half, and it is minimal (less then 5\%) for the down going ones. Note, that this implies $\Delta m^{2} \sin 2 \theta \gg 10^{-3} \mathrm{eV}^{2}$ in order for the weak damping limit to be applicable.

The limits on this decoherence mechanism from terrestrial experiments are rather weak. The existing experiments have a baseline of no more than about $1 \mathrm{~km}$. Hence Eq. (2) predicts that the total transition probability is less then $10^{-3}$ if $\tau$ does not depend on energy. This is consistent with the current bounds from disappearance $\left(P_{\mu \mu}<0.01\right)$ and appearance $\left(P_{\mu \tau}<0.002\right)$ experiments [7]. Note that in some of the accelerator experiments the neutrino energies are much larger than for the atmospheric neutrinos, e.g., $E \sim O(100) \mathrm{GeV}$ [8]. This seems to indicate that $\tau$ should not rapidly decrease with energy. Recall, however, that if the oscillation length is much longer than the baseline, Eq. (2) is not applicable and the transition probability is much more suppressed. We thus conclude that a strong reduction of $\tau$ with energy is excluded for large $\Delta m^{2}$. For example, from Ref. [8] we conclude that $\tau \propto E^{-n}$ with $n \gtrsim 1$ is excluded for $\Delta m^{2} \gtrsim 50 \mathrm{eV}^{2}$.

Astrophysical constraints can also be used to check some of the general features of the mechanism we have proposed. Big Bang Nucleosynthesis (BBN) constraints disfavor $\nu_{\mu}-\nu_{s}$ mixing. To explain the atmospheric neutrino data we require the $\nu_{s}$ interaction time to be much shorter than that for the standard neutrinos. If this continued to hold unchanged in the early universe, it would cause the $\nu_{s}$ to be thermally populated at the epoch of BBN which is disfavored by the data [9]. However, we cannot rule this possibility out since the $\nu_{s}$ may have exotic interactions that result in a tiny effective mixing angle in the early universe, and moreover the energy dependence of $\tau$ is unknown. 
The Solar neutrinos are primarily electron neutrinos $\left(\nu_{e}\right)$ and hence are not directly related to our case. If, however, the $\nu_{e}$ undergo a similar mixing with $\nu_{s}$, then a decoherence length significantly below $1 \mathrm{AU}$ would lead to an energy independent suppression of the solar neutrino flux in contradiction with the data [10]. Since the solar neutrinos have energies in the $\mathrm{MeV}$ range (compared to $E \sim 1 \mathrm{GeV}$ for the atmospheric neutrino) we can conclude that either $\tau$ decreases with energy, or that the $\nu_{e^{-}} \nu_{s}$ mixing is insignificant.

The best way to rule out our proposal is to observe an oscillation pattern in $(L / E)$ at $\mathrm{Su}-$ perK. At present, the uncertainties in $(L / E)$ at SuperK are large resulting in a smearing out of any possible oscillation pattern. Even with the smearing, the disappearance probability as a function of $E$ for the two cases may be different depending on the energy dependence of $\tau$. For the oscillation scenario, the disappearance probability goes as $E^{-1}$ after smearing. If, for example, $\tau$ decreases with energy as indicated by our discussion of solar neutrinos, the disappearance probability will increase with $E$.

The up coming long baseline neutrino oscillation experiments will be sensitive to much of the preferred parameter space of the decoherence hypothesis. From our considerations earlier in the paper, the preferred range for the mixing parameters is $0.01 \mathrm{eV}^{2} \lesssim \Delta m^{2} \lesssim$ $10 \mathrm{eV}^{2}$ and $0.01 \lesssim \sin 2 \theta \lesssim 0.4$ where the smaller values for $\Delta m^{2}$ are correlated with larger values for $\sin 2 \theta$. Thus, the signal will be different from that predicted by the oscillation explanation of the SuperK data. If $\tau$ is independent of energy, the disappearance will be due to a combination of decoherence and oscillation. If, however, $\tau$ decreases with energy the decoherence effect will be minimal and the signal will be purely due to oscillations.

An important prediction of our proposal is that for $t \gg \tau, P_{\mu \mu}=\frac{1}{2}$. This is in contrast to the oscillation scenario where averaging over many oscillation lengths results in $P_{\mu \mu}=$ $1-\frac{1}{2} \sin ^{2} 2 \theta$ which could a priori have any value from $\frac{1}{2}$ to 1 . While $\tau$ can be fine tuned such that $P_{\mu \mu}$ for the atmospheric neutrinos saturates at a value different from half, in general, this will not be the case. If, when more data becomes available, one finds that the saturation is to a value different from half, it will disfavor the decoherence mechanism.

Before concluding let us remark that one can generalize this decoherence mechanism to massless neutrinos. The general requirements for the decoherence mechanism to work are the presence of a mixing angle between the propagating eigenstate and the "flavor" eigenstate - the state that is measured; and that the oscillation length is shorter than the decoherence length. The first requirement can be met if the decoherence mechanism does not measure the weak flavor but rather some superposition of the muon and sterile neutrinos. The second requirement can be satisfied if the unknown interactions generate an "effective" mass for one of the neutrinos.

To summarize, we propose that it is possible that the deficit in $\nu_{\mu}$ observed at SuperK is not due to the fact that $\nu_{\mu}$ 's oscillate over an Earth diameter, but rather that they have oscillated many times and have lost coherence due to some unknown mechanism over this distance. While this explanation is admittedly speculative, it needs to be ruled out in order for the oscillation hypothesis to be confirmed.

Acknowledgements. We thank N. Arkani-Hamed, J. Learned, R. Plaga, G. Sigl and T. Weiler for helpful discussions. 


\section{REFERENCES}

[1] The Super-Kamiokande Collaboration, Y. Fukuda et al., hep-ex/9807003.

[2] T.J. Weiler, private communication; V. Barger, J.G. Learned, S. Pakvasa and T.J. Weiler, in preparation.

[3] S. Glashow, talk presented at Neutrino98.

[4] Large local neutrino density was invoked for other purposes in R.N. Mohapatra and S. Nussinov, Phys. Lett. B395, (1997) 63.

[5] N. Arkani-Hamed, private communication.

[6] L. Stodolsky, Phys. Rev. D36 (1987) 2273; G.G. Raffelt, "Stars as laboratories for fundamental physics", Chicago Univ. Press, 1996.

[7] C. Caso et al. (Particle Data Group), European Physical Journal C3 (1998) 1.

[8] I.E. Stockdale et al., Z. Phys. C27 (1985) 53.

[9] For a recent review see e.g., A.D. Dolgov, astro-ph/9807134.

[10] See, e.g., J.N. Bahcall, P.I. Krastev and A. Yu. Smirnov, hep-ph/9807216. 